\title{
BIMCERT PROJECT: DIGITAL COMPETENCIES TRAINING FOR A MORE SUSTAINABLE BUILT ENVIRONMENT
}

\author{
Antonio Aguiar Costa ${ }^{(1)}$, Paulo Carreira ${ }^{(1)}$, Paul Mccormick ${ }^{(2)}$, Andrew Hamilton (2), \\ Eduardo Rebelo $^{(2)}$, Barry Mcauley ${ }^{(3)}$, Avril Behan ${ }^{(3)}$, Barry Nielson ${ }^{(4)}$, Gayle Beckett ${ }^{(4)}$, \\ Dijana Likar ${ }^{(5)}$, Angelina Taneva-Veshoska ${ }^{(5)}$, Sheryl Lynch ${ }^{(6)}$, William Hynes ${ }^{(6)}$, Toni \\ Borkovic ${ }^{(7)}$
}

(1) Instituto Superior Técnico - Universidade Lisboa, Av. Rovisco Pais, 1049-001 Lisboa, Portugal.

(2) Belfast Metropolitan College, e3 Building, 398 Springfield Road, Belfast, Northern Ireland.

(3) School of Multidisciplinary technologies, Technological University Dublin, Bolton Street, Dublin, Ireland.

(4) CITB Northern Ireland, Nutts Corner Training Centre, 17 Dundrod Road, Crumlin, County Antrim, Northern Ireland.

(5) Institute for Research in Environment, Civil Engineering and Energy (IECE), Drezdenska, No.52, Skopje, R. Macedonia 1000.

(6) Future Analytics Consulting Ltd, 23 Fitzwilliam Square, Ireland.

(7) Energy Institute Hrvoje Pozar, Savska cesta 163, 10001 Zagreb, Croatia.

\begin{abstract}
The global buildings sector is now responsible for $40 \%$ of final energy consumption as well as accounting for $33 \%$ of energy-related carbon dioxide emissions. This has far resulted in a growing urgency to address energy and emissions from buildings and construction, to meet restrictive 2020 targets as specified by the European Union (EU). To achieve these targets several funding initiatives have been put in place through Horizon 2020 with a focus on BIM, due to it having the potential to rapidly produce energy outputs that enable design teams to analyse and compare the most cost-effective, energy-efficient options.

However, despite the recorded benefits that BIM can bring to the design, there is still a lack of understanding of how it can be used on site by the supply chain to impact energy savings directly. In order to address this industry-wide concern a Multi-International consortium bid, won and then launched the Horizon 2020 Energy BIMcert project in March 2018 with the goal to educate all areas of the supply chain in the use of BIM, to achieve better energy efficiency during the design, construction and ongoing maintenance of an asset. An online platform was launched in 2019, which delivers blended training that combines theory, practice, and eLearning to enable workers to train more effectively. This paper will present the project and discuss some of its major results.
\end{abstract}




\section{Introduction}

The exploitation and utilisation of energy resources have caused severe ecological and environmental problems, including the production of emissions that contribute to global warming (Enshassi et al., 2018). The construction industry consumes up to $50 \%$ of mineral resources excavated from nature, generates about $33 \%$ of $\mathrm{CO} 2$ present in the atmosphere and is responsible for $40 \%$ of total global energy through both construction and operational emissions (Ajayi et al., 2016 and Zhou and Azar, 2018).

The realisation that Architecture, Engineering and Construction (AEC) practices now face globalisation, sustainability, and environmental concern, as well as ever-changing legislation requirements and new skills needed for the information age, has resulted in technologies such as Building Information Modelling (BIM) becoming a key enabler in navigating these concerns (Jaradat, 2014). BIM can be defined as a modelling technology and associated set of processes to produce, communicate, and analyse building models (Sacks et al., 2018). BIM provides an opportunity for the Architectural, Engineering, Construction, and Operation (AECO) industry stakeholders to evaluate possible solutions and identify potential problems of the final product before the start of actual construction (Badrinath et al., 2016).

However, changing from traditional practices to BIM requires a shift not only in the technology used but also in the way design and construction teams work together (Shelbourn et al., 2017). To achieve the associated benefits that are accustomed to BIM several existing challenges to Information and communications technology (ICT) utilisation in construction site management must be overcome which include a lack of knowledge, skills and competence, depth of understanding of decision-makers and low ICT literacy (Ozumba and Shakantu, 2017). This BIM movement has also resulted in a clear, direct, and automatic impact upon engineering education systems (Jäväjä and Salin, 2014).

To assist in overcoming these barriers, to reach European Union (EU) energy-related targets some funding initiatives have been put in place through Horizon 2020 with a focus on BIM, as a result of it having the potential to rapidly produce energy outputs that enable design teams to analyse and compare the most cost-effective, energy-efficient options. Such an initiative is the Energy BIMcert project, which aims to educate all areas of the supply chain in the use of BIM, to achieve better energy efficiency during the design, construction and ongoing maintenance of an asset.

The BIMcert $\mathrm{H} 2020$ project set out to design, develop and trial a series of training interventions using digital technology and improved blended techniques to support, enhance and maximise the impact of energy-efficient skills at all operational levels within the modern construction industry. The project developed an accessible portal for training the middle tier of the construction industry supply chain workers. This primary aim of the portal is to assist these workers to improve their BIM skills, ultimately leading to better collaborative working resulting in more energy-efficient, sustainable buildings and increased quality throughout the entire construction supply chain. The long-term goal of the project is to enable participants to go beyond their current non-integrated construction functionality to an optimum digitally-enabled energy efficient position where they can employ all the IT-enabled functionality and the BIM tools, processes, services and products to deliver near-zero carbon construction and renovation. 


\section{Methodology}

BIMcert project was structured into eight work packages, planned to guarantee the integration across all developments (Figure 1).

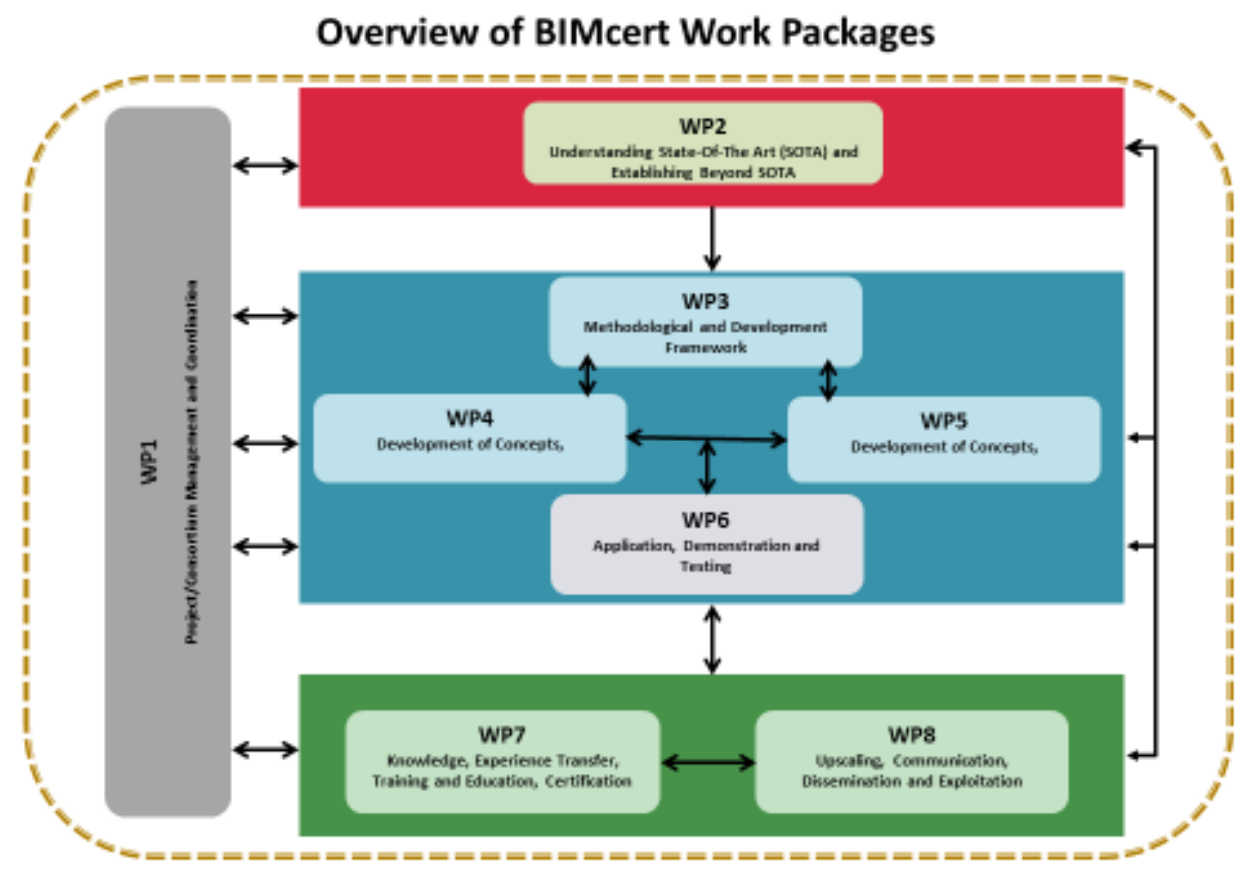

Figure 1: Project Model of BIMcert Work Packages.

The consortium started by establishing a detailed process in determining the training methodologies and associated curriculum. An initial pan European extensive survey of the industry was undertaken to ascertain the current level of BIM maturity, knowledge and understanding within Built Environment practitioners and academia and to establish current standards of sustainable design and construction practice. The results from the survey have been cross-referenced with five workshops within the project stakeholders' jurisdictions. In parallel, it was conducted a literature review of the current global status of BIM regarding education and what pedagogical methodologies are applied to deliver these courses.

The results from the survey and workshops were used to establish the training courses that bestmatched industry needs, supporting the production of a rolling Matrix of Concepts and Methodologies aligned to best practice for knowledge transfer. Both the initial findings for the suggested training courses and Matrix of Concepts and Methodologies have been tested through a series of Reality Check workshops. The outcomes from the Reality Check workshops resulted in the establishment of the final training methodologies, descriptors including learning outcomes, suggested syllabi, and delivery details.

\section{Industry and partners positioning}

One important initial step was to understate the maturity of the industry in terms of BIM and its positioning regarding BIM implement BIMcert aimed to collate the current position of the 
five partner countries concerning BIM/Digital readiness within the construction sector and identify the learning mechanisms that would have the most impact in developing BIM/Digital skills. The methods employed to pursue this objective consisted of a blend of:

- Online survey;

- Initial workshops to provide qualitative feedback;

- Further workshops to check understanding and efficacy of the data gathered and conclusions reached.

The delivery relied on the input of all the partners in each of the participating countries. This process was instrumental in "binding" the project team (Figure 2).

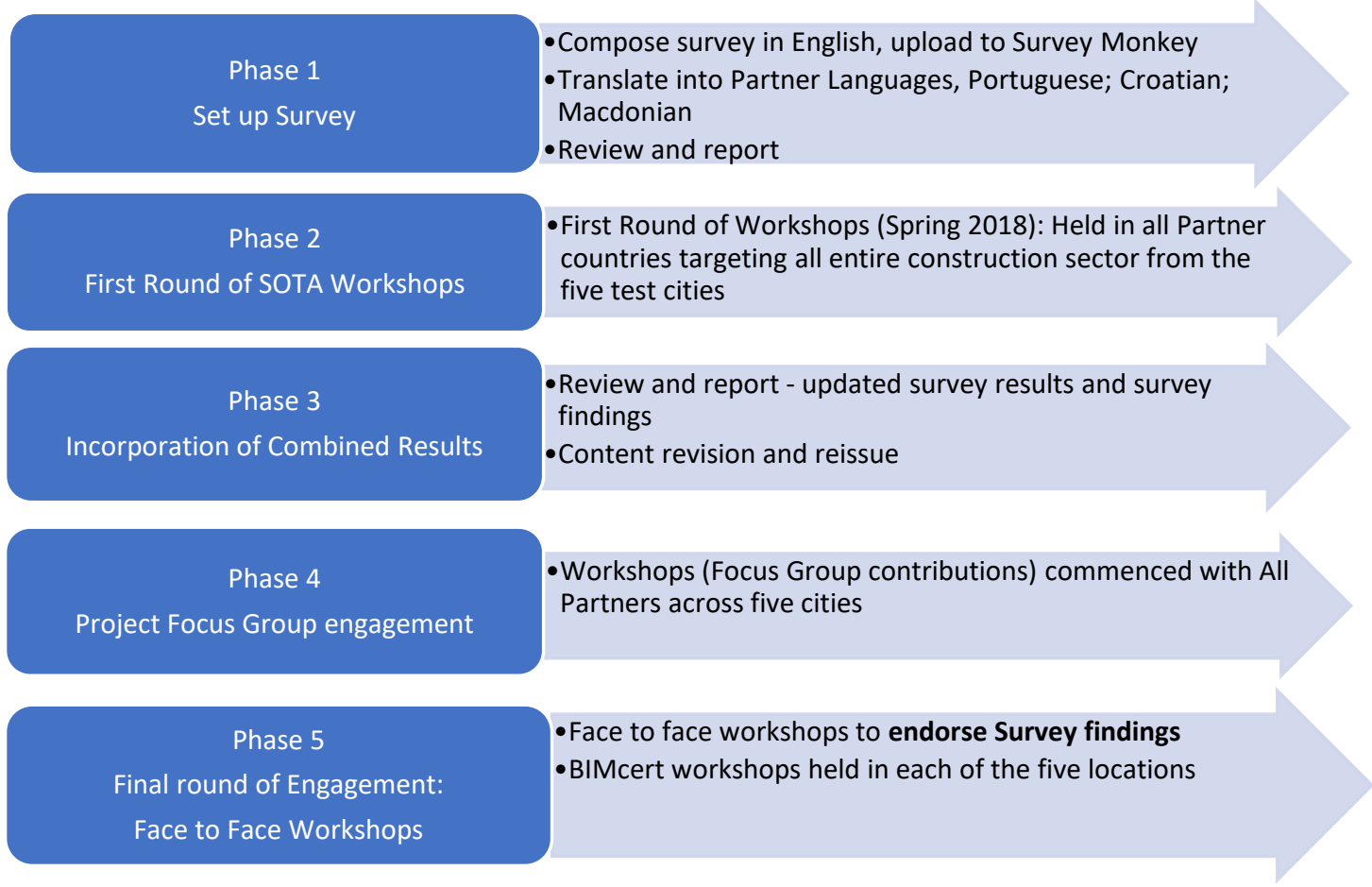

Figure 2: Industry oriented developments.

\subsection{BIMcert Digital Platform}

Workpackage 3 (WP3) developed the BIMcert digital platform by implementing a Moodle framework and several tailor made gamification features within the learning environment. To get to this result, WP3 studied and mapped the learning process, gathered platform requirements, defined software architecture and specifications, developed the technology system and test the final product.

BIMcert platform answers several challenges. First, the workforce needs to acquire an increasing amount and variety of skills, in a shorter time span. Moreover, students and trainees expect that learning should be engaging and practical with real life-type tasks and rather take the form of discussions and negotiations with the teacher/trainer, in a collaborative exercise. To answer these challenges, WP3 stated, since the beginning, the following objectives for the platform: 
- Improving early and continuous assessment and feedback for trainees, providing a feeling of achievement and involvement;

- Motivating self-study, which must be supported by a structured and organised sharing of contents, in parallel with training tools and hand-on work;

- Clarifying the learning objectives and related learning strategies and their relevance to the industry and the trainees' career;

- Increasing interactivity and collaboration.

In this context, gamification appeared as a valuable instrument to motivate and engage trainees, getting them into a "learning" mission that can result in a "rewarded" progression, recognised by an online community. So, it was decided to implement a hybrid solution, based on a Moodle platform and a tailor-made solution for gamification. This gamification module will integrate with the Moodle framework and, so, upgrade the basic e-learning features of Moodle. The rationale for choosing Moodle rests mostly on two key aspects. The first one is the vast and vibrant existing community around the Moodle that offers guarantees that the platform will have continued support and will not be discontinued. Second, we estimated that developing a learning system from scratch would not be feasible due to the respective cost. Therefore, customising and extending an existing an existing system is more cost effective and leverages features already developed by the Moodle community.

With respect to the architecture of the platform, Moodle is a PHP application with an application core, surrounded by numerous plugins to provide specific functionality. These plugins use the middleware functionality provided by the Moodle Core and by the Moodle UI. Moodle is designed to be highly extensible and customizable without modifying the core libraries. In the scope of this project, we created a number of extensions that consist of PHP code and configuration files that give life to BIMcert LMS platform.

The gamification interface created and the moodle framework implemented, which allows multiple and diversified configurations, require considerable training to be adequately used and administrated. Having this in mind, WP3, prepared four training sessions, with four training hours each, for all the consortium partners. Besides this, there was continuous coaching of all the work developed in the platform by other partners, to guarantee the quality of the final delivery. Additionally, WP3 developed a set of training videos, which were uploaded to youtube and are available for the users who need to manage the platform and use their several features.

- Link to the List of BIMcert Training Sessions videos https://www.youtube.com/playlist?list=PLiU2bcTrSNYYUQ82wiYKw4HQ7ojmF2FQ

This list of videos is adequate for BIMcert platform administrators, managers and eventually, trainers. However, we identified also the need to provide several BIMcert platform presentation videos for the trainees. Having this in mind, we created nine short videos, which present the platform, its most significant features and some of the essential activities to conduct with it. The following Youtube list includes all the nine short videos introducing the BIMcert platform.

- BIMcert platform introduction Short Videos

https://www.youtube.com/playlist?list=PLiU2bcTrSNYYeKZ-UfmBNE6hxexwMcD2z 
The videos created within WP3 were also used to develop a part of a BIMcert course, called "Training 4 Trainers". This course aims to present the BIMcert project and platform to trainers and provide them with the necessary tools for a more active and proactive enrolment.

Testing the platform was an important activity within WP3. Several tests were conducted to analyse the platform performance: security tests, compatibility tests, interoperability tests, portability tests, maintainability tests, and usability tests.

For the usability tests, which were one of the most important, WP3 performed a questionnaire to the users. Care was taken to achieve consistent meaning for all respondents, to avoid ambiguity and unsuitable wording to increase the quality of response.

The questionnaire consisted of a group of eleven questions selected from the ISO 9241 standard from the International Organization for Standardization, covering ergonomics of humancomputer interaction. These questions were chosen to take into consideration five fundamental aspects for a proper interface construction: Design, Functionality, Easiness of use, Learnability and Satisfaction. All questions were designed so that the collected answers could be treated using a Likert scale with five possible values, from 1-"Totally Disagree"; 3-"Not agree nor disagree" to 5-"Totally Agree".

The questionnaire was developed using Google Forms and sent to all the users of the platform. In terms of results, 29 users answered the questionnaire. It is a small percentage of the registered users, which ascend to more than 200 (and growing), but other WPs were also conducting surveys, which might have reduced the participation rate. However, according to Cipriano Forza $^{8}$ (Figure 3 ), the size of the sample depends on the effects and associations under analysis, which in the present case are "large effect" analysis. We do not want to make specific conclusions about each one of the questions, or the reasons behind each question, but to have an overview of the usability of the platform. Thus, a sample of 29 users is acceptable for the undertaken analysis.

\begin{tabular}{lcrrr}
\hline & \multicolumn{2}{c}{ Stat. power $=0.6$} & \multicolumn{2}{c}{ Stat. power $=0.8$} \\
& $\alpha=0.05$ & $\alpha=0.01$ & $\alpha=0.05$ & $\alpha=0.01$ \\
\hline Large effect (e.g. strong association) & 12 & 18 & 17 & 24 \\
Medium effect (e.g. medium association) & 30 & 45 & 44 & 62 \\
Small effect (e.g. small association) & 179 & 274 & 271 & 385 \\
\hline
\end{tabular}

Figure 3: Effect size and statistical power and sample size (Forza, 2002).

In terms of responses, the answers to the eleven questions were very positive. The average results were always above 4, within a Likert scale from 1 to 5 (Figure 4). This result means, in general terms, that the users have a very positive attitude towards the platform usability.

\footnotetext{
${ }^{8}$ Cipriano Forza, 2002, "Survey research in operations management: a process-based perspective", International Journal of Operations and Production Management Vol. 22(2)
} 


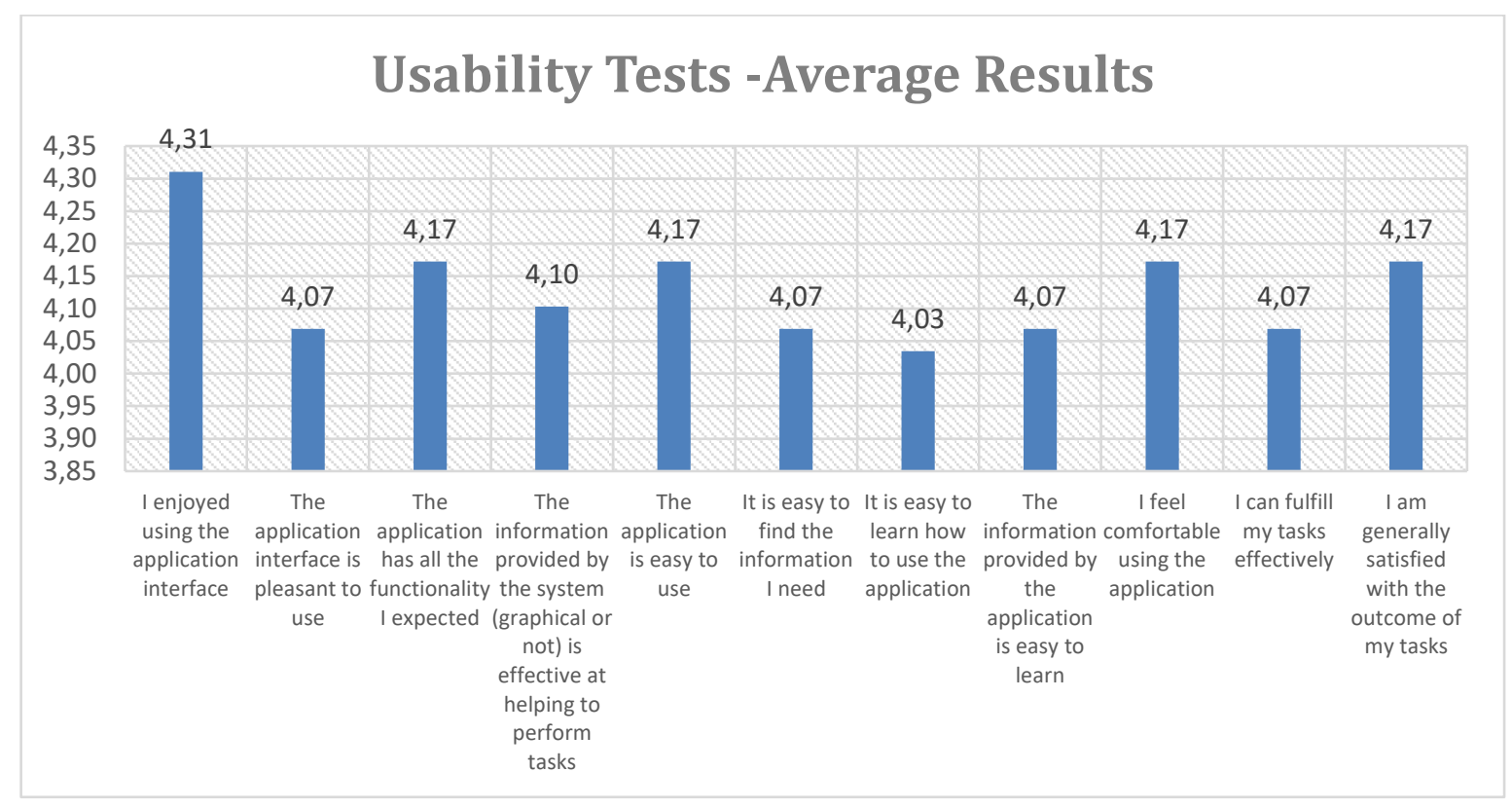

Figure 4: Average results to Usability Tests.

\subsection{Tools and Learning Methodologies}

WP4 undertook a state-of-the-art literature review of current BIM teaching initiatives that represent best practice for knowledge transfer. This exercise identified many potential training methodologies that could be applied to the Energy BIMcert platform. The results from this exercise were cross-referenced with the survey findings from WP 2 and workshop results to ensure that the selected methodology was industry appropriate. The initial findings for the suggested training courses and methodologies were tested through a series of reality check workshops. The outcomes from the reality check workshops resulted in the establishment of the final training descriptors, including learning outcomes, suggested syllabi, methodologies and delivery details. The consortium members decided that the best way forward was to break the development of the curriculum into three strides. Figure 5 identifies the units and courses that best reflect the needs of the industry from the consultation process.

A variety of material from a selection of learning outcomes (LO) from the BIM Ready (Stride 1), BIM Fundamentals (Stride 2A), BIM Principles (Stride 2A), Digital Skills (Stride 2A), and Introduction to Low Energy Building Construction Course (Stride 2C) were developed for testing in Phase 2 of the project. Phase 2 workshops found that while the delivered material was in general well-received, some specific comments were recorded from each workshop. The key thematic findings found that learners would like more applicable case studies, bite-size and shorter learning sections, interactive assessments, clearer learning pathways, and more emphasis on sustainability. Based on the findings from the workshops, each of the partners refined their materials. For the case of the Workshops for Phase 3, it was agreed to create the following modules which represented the LOs from Phase 3:

1. What are BIM (Maturity) Levels?

2. BIM Terms \& Definitions

3. BIM \& Digitalisation Benefits-Overview

4. BIM Dimensions 
5. Intro to BIM tools for Low Energy Building Construction

6. Energy system thinking key principles

7. What is BIM \& digital construction?

8. Intro to BIM Implementation- Impacts in project delivery

9. Digital Skills- Accessing information through the cloud

10. Digital Skills- Accessing information through Portable devices

11. Digital Skills \& Collaboration I-CDE \& File Structure

12. Digital Skills \& Collaboration II - The use of cloud-based storage and portable devices to access and exchange information ( This contained the Digital Skills- Accessing information through the cloud and Digital Skills- Accessing information Portable devices modules)

13. Digital skills \& collaboration III-review BIM models.
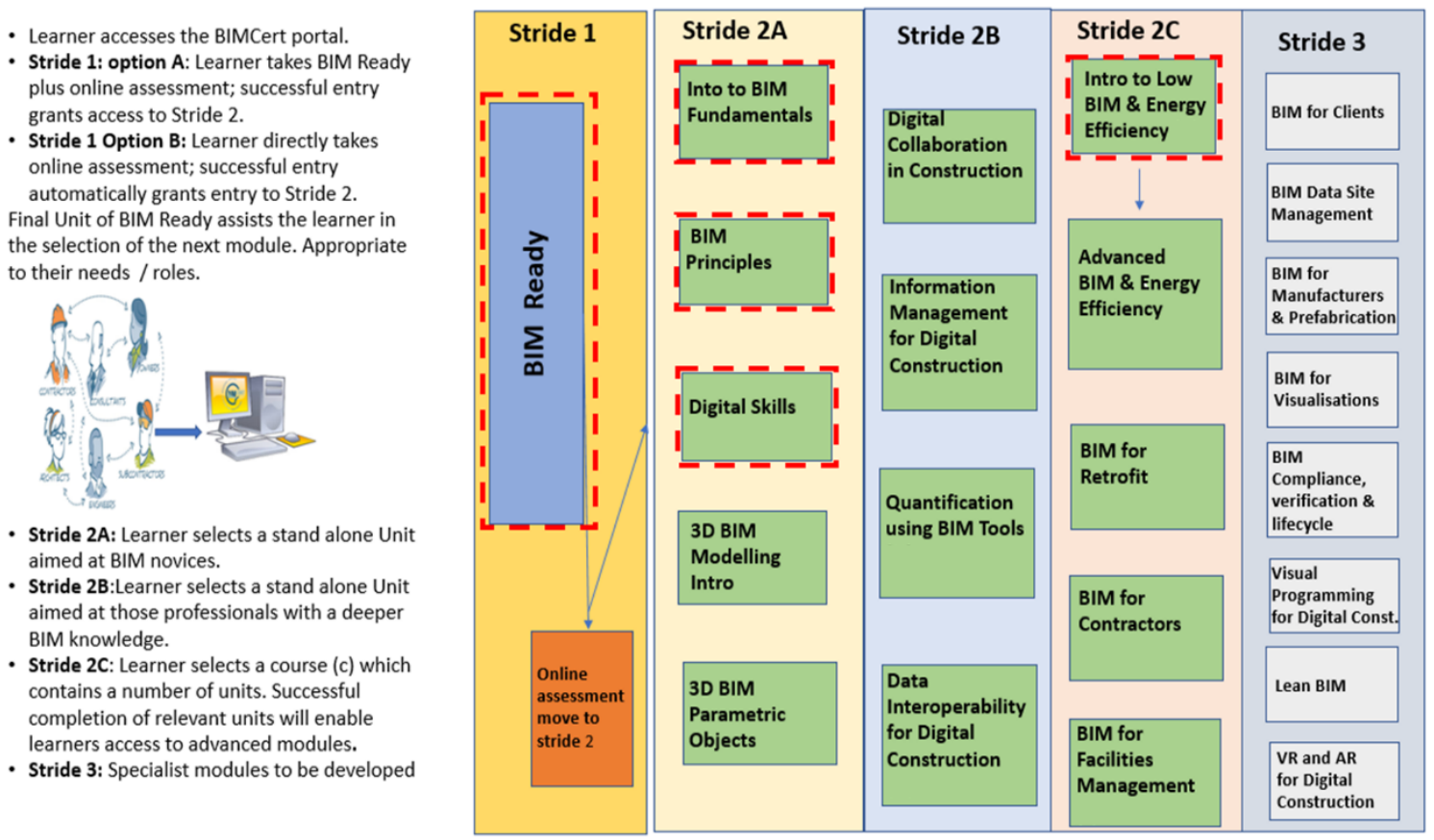

Figure 5: the units and courses that best reflect the needs of the industry.

Each of the modules is required to have an assessment in order to ascertain if the user has passed the module. The assessments were selected on the recommendations from the pilot workshops, as well as international best practice i.e., BuildingSmart. TU Dublin, Belfast Met and IECE worked together to design the assessment content and requirements. It was essential to have a different range of assessments to meet the needs of each of the users. The following assessment methods were used throughout

- Quiz: Once the user finished their learning activities, a quiz was used to assess their knowledge.

- Interactive Quiz. A quiz was built into the tutorial videos, where the user was prompted to stop the lesson and answer the question. 
- Guided Self Learning (Interactive) Assessment: This type of assessment required the student to demonstrate a number of tasks as a result of their lessons from the modules i.e. navigate the model and access the properties and use the mark-up function.

- Illustrated Game Puzzle Self-Assessment: Some of the assessments contained interactive illustrated puzzle games, i.e., drag and drop.

It was agreed amongst the partners that the next set of workshops should be hosted online, with the trainees having access to the BIMcert platform. The webinars aimed to give participants a first-hand experience of using the training platform, where they could access the BIMcert Training materials in the form of modules and training plans. To ensure a smooth transition for both trainers and trainees, two new modules were created titled "Training for Trainers" and "Training 4 Trainees". The "Training 4 Trainees" included 3 sample modules i.e. What are BIM (Maturity) Levels, BIM Terms \& Definitions and Digital skills \& collaboration II-Access BIM models:

\subsection{Methods \& Tools}

Table 1 shows the training methods and appropriate learning materials that were developed for trials and tests of training models and tools, stage 1 (February - April 2019) and stage 2 (September - November 2019).

During the trials, blended learning was applied, progressing from face-to-face towards beyond blended approach, in the first and second round of tests, wherein e-learning was applied. Learners were provided access to the learning and assessment materials via the BIMcert platform. The testing period achieved an end-user engagement increase of 107 people, up by 135\% since the first round of trials (79 participants) in Autumn 2018. There were 97 responses to the Autumn Webinars follow-up surveys, up by 74 responses since Autumn 2018, a 321\% increase in respondents. With the launch of the BIMcert Training Platform, there are 297 registered users (as of January 2020). 87 skilled tradespeople provided their valuable inputs on their outlook on BIM and positively acknowledged the use of the BIMcert Platform for BIM upskilling.

\subsection{Accreditation to EU Standards}

One of BIMcert's goal was to align the project curriculum to recognised standards in order to allow for pathways of certification, or accreditation. These could be inclusion into a National Framework, or European framework, or other possible certification options that would bring recognition to the programme. BIMcert successfully submitted an application to include a new Qualification into the UK educational Framework, to test viability. Following that, further applications were submitted and accepted.

At project completion we have 3 new qualifications in the National Framework, that encompass BIMcert units. The same process could not be replicated in other regions during project timetable, as each country has its own qualification process, with different timeframes which were not compatible with the project durations, or processes that were too depended on third parties to which BIMcert could not access. 
Table 6: Methods and tools

\begin{tabular}{ll}
\hline Unit & \multicolumn{1}{c}{ LO Descriptions } \\
\hline BIM Ready & $\begin{array}{l}\text { An introductory self-study } \\
\text { tool Covering subjects such }\end{array}$ \\
Methodology: & as: What is BIM, BIM \\
Guided Self Study (GSL), & process, BIM maturity \\
Scaffolding & levels, BIM terms, Benefits \\
& \& Barriers, etc. \\
\hline BIM & Define what BIM is and \\
Fundamentals & explain key terminology
\end{tabular}

Methodology:

Web-Based Lecture(WBL),

Instructor-Led Tutoring(ITL),

Scaffolding, GSL

Methodology:

List the benefits \& value of

WBL, ITL, Scaffolding, GSL

a BIM workflow

Materials developed

1. A free self-study and knowledge diagnosis tool to introduce you to BIM and / or provide assessment and recognition of prior knowledge, while getting on track to be ready for BIMcert upskilling and using BIM within your professional role.

1. Instructor Led Live Lecture presentation at all Workshops.

2. Presentation materials bedeveloped to explain the basic principles of BIM and summarise the common terminology associated with BIM. This presentation can be utilised for live web-based delivery (streaming) or recorded as a narrative video.

1. Instructor Led Live Lecture presentation at all Workshops.

2. Presentation material developed to summarise and list the overall benefits of BIM, particularly in relation to specific roles in the construction industry and energy management. This presentation can be utilised for live web-based delivery (streaming) or recorded as a narrative video.

BIM Principles $\quad \begin{aligned} & \text { Explain the context and } \\ & \text { essentials of BIM. }\end{aligned}$

Methodology: essentials of BIM.

1. Instructor Led Live Lecture presentation at all Workshops.

2. Presentation material developed to explain key terms and definitions within BIM, summarise BIM maturity levels, explain the impact of BIM maturity Level 2 requirements for project delivery and Illustrate the benefits of BIM to the construction sector. Lecture presentation on BIM Principles, specifically to address LO1:"Explain the context and essentials of BIM." This presentation can be utilised for live web-based delivery (streaming) or recorded as a narrative video.

Digital Skills
Methodology:
GSL, Mastery Learning (ML),
Case Studies (CS), Narrative
Videos (NV)

Describe the use of digital skills and devices in construction.

1. Online guided self-learning training

2. Materials in form of presentations, narrative videos and case studies and links for GSL, developed to demonstrate how to analyse the use of digital skills and devices in construction.

Methodology:

GSL, Mastery Learning (ML),

Case Studies (CS), Narrative

Videos (NV)

Intro to Low Energy Building Construction System Thinking.

Describe how to use digital skills and devices to access digital infromation

1. Online guided self-learning training

2. Materials in form of presentations and narrative videos developed to demonstrate how to access BIM models and information.

1. Instructor Led Live Lecture presentation at all Workshops.
2. Presentation material developed to demonstrate the key principles of System Thinking. This presentation can be utilised for live web-based delivery (streaming) or recorded as a narrative video. SGL links provided

GSL, WBL, NV, CS

Methodology:

Illustrate how BIM can be WBL, Scafoldding, GSL, NV, utilised in Low Energy PBL
1. Live demonstration of softwre and plug in(s) to show energy assessment/simulation to address the LO6 "Illustrate the use of BIM tools ton reduce energy loss"

2. Developed: course books, presentations, narative videos and two BIM energy models (of a new and of a renovated builing) to enable PBL; SGL links provided 


\subsection{Communication, Dissemination and Exploitation}

During project development, it became apparent that construction chain, blue-collar particularly, respond better to direct communication than online communication. Web statistics confirm a total number of $>6660$ web page unique visits, with $>1586$ users registered for the BIMcert newsletter and training, and more than 1564 users registered on training platform. Twitter, as social media, attracted a lower number of profile followers, but as a network which is more inclined towards quick information exchange, it also generated $>220.000$ impressions of BIMcert information. In combination with Google search, BIMcert topics have been delivered more than 300.000 times to potential market.

\section{Conclusions}

Technology is disrupting the construction Industry. $70 \%$ of construction companies believe that those who do not adopt digital tools will go out of business. The BIMcert project provides construction companies with the key digital transformation skills in BIM is delivering actual impacts on the energy performance of buildings. The project has achieved over $30 \mathrm{GWh}$ of energy savings through a better enabled and equipped workforce.

Projects such as BIMcert have brought us to the threshold of digitalisation. We need to upskill ourselves and our society in order to step through the doorway successfully. Construction sector employees are at the liminal threshold of energy transition and digitalisation. To successfully stimulate the demand for sustainable energy skills we need to nudge and assist employees to adopt digitalisation and apply it in the context of energy skills. We need to focus on the development of new patterns to replace the old ways of learning and forming new patterns that demand, stimulate, encourage, unite and assist.

Follow up projects will need to use digitalisation and the certification of "step by step" competences recognition as accelerator to empower demand for energy skills. BIMalliance, which is a follow up project, will build upon the previous work developed by BIM alliance partners in $\mathrm{H} 2020$ projects (BIM-CERT, BIMEET, BIMplement and NET-UBIEP). Capitalising on the outputs and results of these projects to create and deliver a more complex, complete and integrated training scheme. The alliance seeks to combine the results, frameworks and materials previously developed, continue their evolution as well as deeper and further consolidated actions towards raising the uptake and need of these skills. It is planned that we will develop modules for upskilling and appropriate certification tailored for professionals and other participants (public administration and policy makers, owners, facility mangers) and for different construction activities - new and renovated buildings. Certification will be developed to ensure the identification of new "skills" that should be added in the national qualifications to align them with the new requirements of the building market. This process will not be completed in the life time of the project but we will initiate the process and, we can provide the possibility to include it in EUROPASS curriculum in a standardised way as it happen now with the languages level through the "maturity level" approach.

Despite all the advantages, digitalisation alone cannot stimulate demand. However a unified certification scheme based on recognised curriculum frameworks, covering the widely recognisable, essential learning outcomes while incorporating best practices, and addressing the needs and gaps in the industry comprised of professional modules for all participants, can! - because are the properly skilled users that drive transformation, with digitalisation as a tool. 


\section{References}

[1] Abdirad, H. and Dossick, C. (2016) BIM Curriculum Design in Architecture, Engineering, and Construction Education: A Systematic Review, Journal of Information Technology in Construction, ITcon, Vol. 21 (2016), pg. 250

[2] Aghaegbuna Obinna U. Ozumba, À. O.U. and Shakantu, W. (2017) Exploring challenges to ICT utilisation in construction site management, Construction Innovation, Vol. 18 Issue: 3 pp 1471-4175

[3] Ajayi, S.O. Oyedele, L.O. Kadiri, K.O. Akinade, O.O., Bilal, M., Owolabi, H.A. and Alaka, H.A. (2016) Competency-based measures for designing out construction waste: task and contextual attributes, Engineering, Construction and Architectural Management, Vol. 23 Issue: 4, pp.464-490

[4] Badrinath, A.C., Chang, Y.T and Hsieh, S. H. (2016) A review of tertiary BIM education for advanced engineering communication with visualization, Visualization in Engineering, Iss $4: 9$, pp 2-17

[5] Becker, T., Jaselskis, E. and McDermott, C. (2011). Implications of Construction Industry Trends on the Educational Requirements for Future Construction Professionals. International Conference Proceedings of the 47th Associated Schools of Construction, Omaha, Nebraska, United States, 6-9th April, pp 1-12

[6] Enshassi, A., Ayash, A. and Mohamed, S. (2018) Factors driving contractors to implement energy management strategies in construction projects, Journal of Financial, Management of Property and Construction, Vol. 23 Issue: 3, pp.295-311

[7] Guy, R. and Lownes-Jackson, M. (2013) Web-based tutorials and traditional face-to-face lectures: a comparative analysis of student performance, Informing Science and Information Technology, Vol. 10, pp.241-259.

[8] Jäväjä, P. and Salin, j. (2014) BIM Education: Implementing and Reviewing "OpeBIM" BIM for Teachers, Proceedings of the Computing in Civil and Building Engineering, Orlando, Florida, June 23-25, pp 2151 - 2158

[9] Jaradat, S. (2014) Educating the Next Generation of Architects for Interdisciplinary BIM Environments, Proceedings of the AAE International Conference on Architectural Education: common currency, University of Sheffield - 3rd to 5th September, pp 127 -135 